\title{
¿Pensar el cuerpo femenino como diálogo de saberes?
}

\author{
Andrea de Lourdes Castillo-Muñoz' (iD 0000-0001-7893-7651 \\ Gloria Mora-Guerrero' (iD 0000-0002-2391-4353 \\ 'Universidad Católica de Temuco, Temuco, IX Región, Chile. \\ 4780000 - dirinves@uct.cl
}

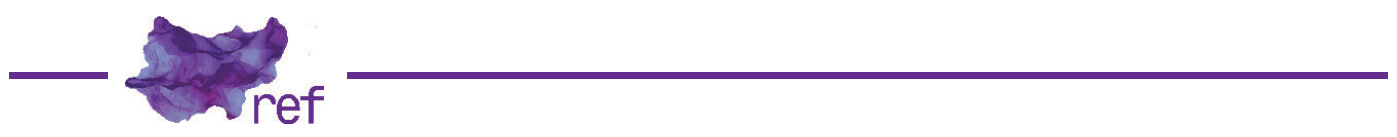

Resumen: La investigación buscó describir los significados que, en torno a la menstruación, producen las feministas de un colectivo en el sur de Chile, y la relación entre estos significados y la cultura regional indígena, mapuche, que forma parte de los referentes simbólicos de las mujeres para la producción de significados corporales. Fue un estudio cualitativo, basado en la teoría fundamentada, con enfoque de género y perspectiva intercultural. Por medio de entrevistas y un grupo de discusión, los resultados mostraron múltiples significados en torno a lo menstrual, originados por las distintas posiciones históricas y espaciales de las mujeres, con la cultura mapuche. Las conclusiones problematizan el feminismo menstrual, al mostrar sus limitaciones para reconocer las diferentes experiencias corporales entre las mujeres del hemisferio sur y resistir la colonialidad simbólica occidental.

Palabras clave: menstruación; feminismo menstrual; interculturalidad; pueblo Mapuche; Chile.

\section{Thinking the female body as a dialogue of knowledges?}

Abstract: The meanings of menstruation for a women's feminist collective living in the south of Chile and its relation to the Mapuche indigenous culture were described. The research method was based on the grounded theory and developed from a gender approach and an intercultural perspective. Focus groups and interviews were conducted to identify the beliefs about menstruation, which are assumed to be produced according to the historical and spatial perspective of each woman regarding the Mapuche culture. Conclusions acknowledge the limitations of menstrual feminism, in relation to the diversity of corporal experiences between women of the southern hemisphere and the different ways of resisting symbolic western coloniality.

Keywords: Menstruation; Menstrual feminism; Interculturality; Mapuche culture; Chile.

\section{Pensar o corpo feminino como diálogo de saberes?}

Resumo: A pesquisa buscou descrever os sentidos que as feministas de um coletivo do sul do Chile produzem em torno da menstruação, e a relação entre esses significados e a cultura indígena regional mapuche, que faz parte dos referentes simbólicos das mulheres para a produção de sentidos corporais. Trata-se de um estudo qualitativo, com base na teoria fundamentada, com uma perspectiva de gênero e uma perspectiva intercultural. Por meio de entrevistas e de um grupo de discussão, os resultados evidenciaram múltiplos significados em torno do menstrual, originados pelas diferentes posições históricas e espaciais das mulheres, com a cultura mapuche. As conclusões problematizam o feminismo menstrual, mostrando suas limitações no reconhecimento das diferentes experiências corporais entre as mulheres do hemisfério sul e na resistência à colonialidade simbólica ocidental.

Palavras-chave: menstruação; feminismo menstrual; interculturalidade; cultura indígena Mapuche; Chile. 
Después de la reivindicación de los derechos civiles, a comienzos del siglo XX, y de la democracia y los derechos humanos en los años setenta, el feminismo latinoamericano se volcó a la lucha contra el patriarcado y las normas de género (Julieta KIRKWOOD, 1986; Sandra PALESTRO, 1991; Claudia MORA; Marcela RíOS, 2009). Las feministas de entonces conceptualizaron que, debido a las normas patriarcales y los roles de género, el cuerpo femenino fue considerado durante siglos como inferior al cuerpo de los hombres (Miren GUILLO, 2013). En la actualidad, ciertas feministas, que pueden llamarse feministas menstruadoras, han ido más allá al señalar que la conceptualización negativa del cuerpo de las mujeres tiene su nudo en la estigmatización de la menstruación la que, en la cultura patriarcal, fue considerada como evidencia de la suciedad e inferioridad de sus cuerpos (GUILLO, 2013). En reacción a ello, las feministas menstruadoras, usualmente agrupadas en colectivos, han buscado referentes simbólicos entre las culturas indígenas que les permitan reconceptualizar la menstruación por fuera de la inferioridad con la que se simboliza en la cultura patriarcal (GUILLO, 2013). En la Araucanía, al sur de Chile, las feministas menstruadoras han buscado estos referentes en la cultura indígena mapuche, la que forma parte del referente simbólico regional (Lucy KETTERER, 2011). En este contexto, la investigación se centró en el feminismo de reivindicaciones menstruales en la Araucanía, especialmente, en las producciones de significados menstruales que realiza el colectivo feminista Círculo Hijas de la Luna Wall Mapu.

El Círculo Hijas de la Luna Wall Mapu (en adelante, el Círculo) es un colectivo feminista conformado por mujeres, algunas de ellas chilenas y otras autoadscritas mapuche, grupo indígena de la zona. Su nombre, Wall Mapu, alude al nombre histórico utilizado por el pueblo mapuche para indicar su territorio desde el Bío Bío al sur de Chile. Las integrantes del Círculo dicen retomar elementos ancestrales de la cultura mapuche para reinterpretar sus vivencias menstruales, de tal manera de reconectarse con su ciclo natural menstrual, que sería similar al ciclo lunar, lo que a su vez las llevaría a vivir la menstruación con naturalidad y sin considerarla sucia. No obstante, más allá de estas declaraciones experienciales de las mujeres feministas del Círculo, no se encontraron estudios ni en Chile ni en Latinoamérica que den cuenta de cómo tiene lugar este proceso de resignificación corporal que supone un encuentro entre la lucha feminista y la recuperación de elementos culturales indígenas.

La presente investigación aborda este vacío en la literatura. Para ello, las preguntas del estudio refieren a cuáles son los significados corporales que construyen las mujeres feministas del Círculo, específicamente, sobre la menstruación, y qué elementos refieren que retoman de la cultura mapuche para construir estos significados. Se trató de un estudio con enfoque cualitativo y diseño de teoría fundamentada con perspectiva de género (John CRESWELL, 2014; Sandra HARDING, 1996) e intercultural (Diana DE VALLESCAR, 2010). La epistemología de base fue la hermenéutica postmoderna (Hans-Georg GADAMER, 1997), aplicada a los diseños de teoría fundamentada (Udo KELLE, 2005).

La información se obtuvo mediante entrevistas semiestructuradas (Orfelio LEÓN; Ignacio MONTERO, 2015) y un grupo de discusión. Se entrevistó a nueve integrantes del Círculo, considerándose diversidad en la muestra en cuanto a auto adscripción étnica, mapuche o no mapuche, y años de participación en la agrupación. En cuanto al grupo de discusión, éste se conformó por mujeres mapuche sin participación en el Círculo, mujeres de la región no auto adscritas mapuche y también sin participación en el Círculo y, por último, por mujeres autoidentificadas feministas, autoadscritas mapuche o no mapuche, y que eran integrantes del Círculo. Las entrevistas permitieron generar información sobre los significados en torno al cuerpo y la menstruación que construyen las integrantes del Círculo. Por su parte, el grupo de discusión permitió ahondar en los elementos que las integrantes del colectivo Hijas de la Luna refieren que retoman de la cultura mapuche para la construcción de estos significados, a la vez que las confrontó con la visión de otras mujeres que, desde diferentes posiciones, reconstruyen la cultura indígena ancestral regional y el lugar que en ella ocupa el cuerpo feminino.

Posterior al análisis de los datos, se realizó un chequeo de los resultados con las participantes del Círculo, como medio de validación de la investigación. Todas las entrevistadas accedieron a participar voluntariamente, lo que se ratificó por medio de la firma de un documento de consentimiento informado. Los resultados mostraron tres procesos socioculturales a partir de los cuales se dio cuenta de los procesos de significación sobre el cuerpo y la menstruación, que tienen lugar en el colectivo de estudio desde la experiencia de sus integrantes. Estos procesos son: uno, la toma de consciencia del castigo corporal; dos, la transformación del cuerpo castigado; y, tres, la transcendencia de lo corporal entre mujeres. Cada uno de ellos se explica en el apartado correspondiente a los resultados. El análisis de las categorías conceptualizadas por las autoras se apoyó en el uso del programa OpenCode versión 4.02.'

1 Software fácil de usar, levantando categorías de forma inductiva y sin cruce de ellas, agrupándolas según su similitud de forma jerárquica. Permite distinguir unidades de análisis y observación relevantes para un tema permitiendo construir el conocimiento y saber organizarlo para una adecuada sistematización. 
Previo a la presentación de los hallazgos, se presenta un recuento de las reivindicaciones que el feminismo ha hecho con relación al cuerpo de las mujeres en Occidente y, en específico, en el contexto latinoamericano, donde la reivindicación de lo femenino intersecta con lo racial y colonial. Posteriormente, se describe la propuesta del activismo menstrual como acción simbólica que representa la tradición del feminismo occidental en su encuentro con las culturas indígenas, en este caso de estudio, la cultura mapuche.

\section{La reivindicación corporal del feminismo latinoamericano: el aporte del feminismo menstrual}

En Occidente, el feminismo se relacionó con la reivindicación corporal en la medida que cuestionó la ubicación del cuerpo femenino como inferior al cuerpo masculino (GUILLO, 2013). Aunque, en particular, fue el cuerpo de las mujeres por oposición al de los hombres el que se inferiorizó, en lo general, fue lo corporal lo que se menospreció desde que se concibió al cuerpo como separado del espíritu o alma y se le redujo a un objeto de explicación racional (René DESCARTES, 1641), despreciable, desalmado e impuro (Stella SOENGAS; Silvia ZAMORANO, 2009). El dualismo y el desprecio del cuerpo provino del equívoco de los pensadores griegos que entendieron el cuerpo como el soma griego (el cuerpo como objeto) y no como carne que se refería a la basar hebrea (el cuerpo como un rostro) (Enrique DUSSEL, 2007). Maurice Merleau-Ponty (1945), filósofo posmoderno, cuestionó el dualismo cartesiano cuando consideró el cuerpo como un organismo sexuado, inacabado y afectado por su entorno, pero no llegó a concebirlo desde la diferencia sexual. No fue sino hasta en época muy reciente cuando la sexualidad se vio como un modo de ser en el mundo, que se da en las relaciones con el Otro, donde el Otro se ve como un rostro, no como un objeto; fue, entonces, cuando se pudo concebir el cuerpo sexual de las mujeres como un otro sexual distinto (DUSSEL, 2007).

En la cultura occidental, el feminismo reivindicó el cuerpo femenino, primeramente, bajo dos perspectivas: la del feminismo de la diferencia y la del feminismo de la igualdad, ambos coincidentes en su visión del cuerpo como sexuado. El feminismo de la igualdad consideraba el cuerpo sexualmente determinado, pero con una mente neutra (Mari Luz ESTEBAN, 201 1; Marta LAMAS, 2000), mientras que el feminismo de la diferencia concibió el cuerpo como algo fundamental para construir la identidad femenina, como principio ordenador del deseo, lo simbólico y el poder de las mujeres. En la postmodernidad, el feminismo se alejó de ambas visiones para mostrar lo corporal más allá de lo sexual, como producto de la cultura o la política, que superaba la mera diferencia sexual entre hombre/mujer. En este punto, teóricas como Amorós, Fraser y Butler sostuvieron que no es que una cultura actúe sobre una naturaleza dada, como el cuerpo de las mujeres, sino que el cuerpo mismo se inserta en un discurso formativo o performativo (Judith BUTLER, 2002). No obstante, el feminismo postmoderno siguió concibiendo el cuerpo como algo abstracto ya que, a pesar de reconocer la diversidad entre las mujeres, la herencia occidental de la que provenían la mayoría de sus autoras les impidió dar cuenta de la opresión social y cultural que viven las mujeres en sus contextos concretos (Asunción OLIVA, 2015). ${ }^{2}$

Esta última visión fue introducida tardíamente por el pensamiento y el feminismo latinoamericano que, desde su visión decolonial, reivindicó el cuerpo como racializado y sexualizado. En efecto, el feminismo decolonial denunció que la colonización implicó también una racialización de los cuerpos indígenas, que fueron entendidos como salvajes e inacabados, cuerpos sin alma y lugares de lo maligno (Cecilia SALAZAR, 2006). Para el feminismo decolonial, la reivindicación del cuerpo femenino en Latinoamérica debía asumir que, en esta zona del mundo, ser mujer se entrecruzaba con la colonialidad y la raza (María LUGONES, 2008; Rita SEGATO, 2010) y que este entrecruzamiento había permitido que los cuerpos de las mujeres indígenas no sólo fueran vistos como inferiores, sino también como controlables, explotables y medios de satisfacción del apetito sexual de los hombres blancos (DUSSEL, 2007). De este modo, el feminismo decolonial cuestionó el etnocentrismo de los feminismos occidentales, revelando que fueron incapaces de reconocer la etnicidad que la colonialidad impuso a los cuerpos de las mujeres indígenas (Aida HERNÁNDEZ, 2001).

Respecto a la inferioridad que pesa sobre el cuerpo femenino, las feministas decoloniales en Latinoamérica se propusieron subvertirla desde el encuentro con lo indígena, de manera que no sólo se pudiera superar la dicotomía alma/cuerpo, sino también la etnicidad y la colonialidad (Julieta PAREDES, 2013). Al respecto, Vicenta Mamani (2014), teóloga feminista aymara, señaló que la descolonización de los cuerpos de las mujeres latinoamericanas e indígenas tendría lugar a medida que ellas se autovaloraran y fortalecieran su

2 Son referentes del feminismo postmoderno Judith Butler, Beatriz Preciado, Nancy Fraser, Julia Kristeva, Jane Flax y Teresa De Lauretis. 
autoconfianza. Mientras que, por su parte, desde el feminismo negro, Ochy Curiel y María Galindo (2015) señalaron que la decolonización de los cuerpos de las mujeres se lograría a condición de que lo corporal pudiera pensarse en términos de la no apropiación ni control por la raza o clase. La lucha de este feminismo es la reivindicación de la deshumanización de la mujer negra, cuestión que el feminismo blanco no daría espacio dificultando hablar de feminismo latinoamericano.

Herederas de las discusiones anteriores, en los últimos años, las feministas menstruadoras se propusieron retomar elementos culturales indígenas como ejercicio contrahegemónico y de resignificación corporal en la cultura occidental. Para ellas, el cuerpo menstruante fue entendido como un cuerpo político, subversivo, que buscaba reconstruirse en su relación con la salud y la identidad femenina (GUILLO, 2013). Según Karina Felitti (2015), las feministas menstruadoras dejaron atrás la bandera feminista de los sesenta, "lo personal es político", y avanzaron hacia una nueva bandera, que podía llamarse la "política de lo personal", o la política menstrual. ${ }^{3}$ Esta última implicaba asumir, por un lado, que los cuerpos eran producto de representaciones culturales que se concretaban como conductas encarnadas de forma individual y colectiva (ESTEBAN, 2011); y, por otro, que los cambios eran posibles porque los sujetos podían descifrar y resignificar tales representaciones, no sólo desde su posición personal, sino desde su posición cultural y social (María de la Luz HURTADO, 2010).

En este contexto, los cambios buscados por las feministas menstruadoras se opondrían a la cultura occidental en dos dimensiones: una, en la reconciliación con la menstruación, por medio de eliminar el tabú y la vergüenza (Merskin DEBRA, 201 1; Chris BOBEL, 2010); $y$, dos, en una resistencia frente al sistema capitalista colonial, por medio de cuestionar la necesidad de los llamados productos de cuidado femenino, tales como toallas, tampones y anticonceptivos, y promover su cambio por productos alternativos saludables, no contaminantes y menos costosos económicamente (BOBEL, 2010). Motivadas por este horizonte, las feministas menstruadoras retomaron concepciones indígenas donde lo menstrual parecía asociarse con la salud, la sexualidad, la vida ética, la sociedad y la tierra (Jorge HIDALGO; Nelson CASTRO, 2010; CEPAL, 2014), lo natural (Ziley MORA, 2006), lo sagrado y lo benéfico (Ximena CARREÑO, 2011 ), la fertilidad (Margarita CALFíO, 2012), la complementariedad de los sexos (Ana Mariella BACIGALUPO, 2003-2004, 2013; Ximena LEVIL, 2015), el paso de las mujeres a la vida fértil (Miguel Ángel ALARCÓN, 2005) y el reforzamiento de lo femenino como alimento de deidades (HIDALGO; CASTRO 2010). En suma, por medio de retomar estas significaciones, que catalogaban como ancestrales, las feministas menstruadoras se proponían reconstruir sus cuerpos para lograr una doble liberación, tanto de la inferioridad femenina, como de la colonialidad capitalista.

A pesar de lo anterior, con este tipo de activismo las feministas menstruadoras corrieron el riesgo de apropiarse arbitrariamente de elementos pertenecientes a las culturas colonizadas, para ajustarlas a su propia liberación (DEBRA, 201 1); o, en otras palabras, corrieron el riesgo de convertirse en un nuevo proyecto occidental de colonización indígena. Además, a pesar de que las feministas menstruadoras han buscado lo ancestral en las culturas indígenas, han parecido obviar que los significados que tenía el cuerpo femenino en estas culturas cambiaron a partir de la colonización (LUGONES, 2008; SEGATO, 2010). En efecto, en el mundo indígena y en particular en el mundo mapuche, la menstruación significa la pertenencia a la línea femenina del universo y la relación estrecha entre las mujeres y la tierra a quien conocen (sus ciclos de fertilidad) y a quien ofrendan su sangre; es una bendición para el pueblo porque indica reproducción para el futuro (Loreto GARCíA; Soledad ROJAS, 2014), y constituye el referente cultural de las Hijas de la Luna, participantes de este estudio. Empero, los significados prehispánicos no peyorativos sobre la menstruación sufrieron transformaciones. De esta manera, en el sistema colonial lo menstrual empezó a concebirse como enfermedad dolorosa, maldición (MORA, 2006; Sonia MONTECINO, 1984, CALFÍO, 2012; BACIGALUPO, 2013; CARREÑO, 2011), falta moral o impureza (DUSSEL, 2007) o, simplemente, asociado al mal o a lo kalku (MONTECINO, 1984). Si bien no se encontraron suficientes estudios que den cuenta de en qué medida el feminismo menstrual reconoce la historia del cuerpo y la menstruación en las culturas indígenas, por otra parte, sí es posible suponer que en la medida en que las feministas menstruadoras no reconozcan dicha historia, estarían reproduciendo la limitación etnocentrista del feminismo occidental cuando usa a las mujeres indígenas sólo como un medio para reificar el universalismo de la mujer blanca (CURIEL; GALINDO, 2015).

\footnotetext{
${ }^{3}$ Hay un cambio significativo entre lo personal es político, vale decir que lo que hago personalmente en la vida diaria de las mujeres es político e influye en la transformación social y la política de lo personal que apunta a la dimensión política de los cuerpos y la vida; esto es cómo las mujeres se construyen desde lo sexual y corporal. Las relaciones son actos políticos de liberación, es ir más allá de lo personal o ámbito doméstico sin reducir lo político a lo personal.
} 
La presente investigación se ubicó en esta discusión por medio de estudiar el feminismo menstrual en la Araucanía para indagar en los significados corporales sobre la menstruación que construyen las mujeres feministas del Círculo, así como en los referentes simbólicos de la cultura mapuche que dicen retomar para la construcción de estos significados. La intención fue aportar a la reflexión sobre la posibilidad de establecer un diálogo entre el feminismo menstrual y lo indígena, y las implicaciones que dicho diálogo tendría para las mujeres. Como sostienen García y Rojas (2014), fruto de este diálogo podría lograrse la liberación de la identidad femenina mapuche sostenida en el mapuche kimün (saber ancestral) devaluado por la sociedad chilena, pero a condición de que las feministas menstruadoras asuman el reto de descentrar la razón occidental y reflexionar críticamente sobre el proceso de descolonización (Catherine WALSH, 2012). El diálogo intercultural entre el feminismo menstrual y las culturas indígenas, a fin de no ser un instrumento de colonización, deberá implicar la reelaboración o recreación de la cultura (DE VALLESCAR, 2010) o constituirse en un diálogo entre diferencias culturales (Raúl FORNET-BETANCOURT, 1988), pero sin que una cultura se sobreponga a la otra para sus propios fines. En el apartado siguiente, se presentan los resultados del estudio.

\section{Pluralidad de cuerpos menstruantes: ¿camino hacia el diálogo intercultural?}

En relación con las preguntas sobre los significados menstruales que construyen las mujeres feministas del Círculo y su relación con elementos de la cultura mapuche, el análisis mostró tres procesos corporales que experimentan las participantes de este colectivo: uno, la toma de consciencia del castigo corporal que han vivido como mujeres; dos, la transformación de sus cuerpos sangrantes; y, tres, la experiencia de trascendencia corporal entre mujeres. Los tres procesos o categorías de análisis fueron consensuadas entre las participantes y las autoras y pueden entenderse como parte de un macroproceso, experimentado por las integrantes del Círculo, y que aquí se ha llamado pluralidad de cuerpos menstruantes: ¿camino hacia diálogo intercultural? Este último proceso pretende describir, primero, la pluralidad de experiencias y significados que tiene la menstruación entre las integrantes del Círculo; y, segundo, las posibilidades que ellas tienen de entablar un diálogo intercultural entre el feminismo menstrual y la cultura mapuche regional.

De acuerdo con los resultados, los anteriores procesos experienciales de las integrantes del Círculo, las autoras los relacionan con tres posiciones que pueden describir su acción al interior del colectivo. La primera, se trata de una posición que puede llamarse feminismo chileno/occidental; ésta constituye la posición dominante entre las activistas del Círculo. Es ocupada mayormente por mujeres chilenas no autoadscritas como mapuches, que se relacionan con esta cultura por medio de lecturas $u$ otros elementos de tipo discursivo. Los significados que las mujeres producen desde esta posición predominan en los diálogos al interior del Círculo y dan mayormente sentido a las acciones que allí se realizan; se podría decir que es la posición desde la que se enuncian significados con autoridad y por medio de los cuales se ejerce el liderazgo. En términos generales, los significados enunciados desde esta posición no aluden al proceso colonizador mapuche ni reconocen un cuerpo racializado femenino.

La segunda posición dentro del Círculo se ha denominado feminismo mapuche/chileno y corresponde al lugar, por lo general, ocupado por mujeres que se autoadscriben como mapuche y mayormente radican en zonas urbanas, aunque mantienen vínculos con sus comunidades de origen. Desde esta posición, ellas producen significados que dan cuenta con vaguedad de la historia racializada de las mujeres mapuches y sus demandas históricas como pueblo. Por este motivo, con frecuencia estas mujeres asumen los significados dominantes que se articulan desde el feminismo chileno/occidental.

Por último, la tercera posición dentro del colectivo de estudio se ha llamado feminismo apegado a lo mapuche, lugar que corresponde, por lo común, a las mujeres participantes del Círculo que están vinculadas a las prácticas y creencias mapuches, primero, porque se autoadscriben como mapuches y, segundo, porque están fuertemente vinculadas a sus comunidades y territorios de origen. Los significados producidos desde esta tercera posición reconocen el sistemático colonialismo del que ha sido víctima el pueblo mapuche y, desde este lugar, enuncian sus demandas de emancipación personales y como miembros de la cultura mapuche.

En el siguiente apartado, se exponen los significados que las feministas del Círculo producen desde cada una de estas posiciones. Tales significados se organizan a partir de los tres procesos corporales que las mujeres experimentan durante su participación en el colectivo. Al finalizar la exposición, se discuten los hallazgos y se concluye en torno a los alcances de la investigación. 


\section{Toma de consciencia del castigo corporal}

Según las integrantes del grupo, durante su participación en el Círculo, ellas constatan o toman consciencia de que, como mujeres, han sufrido el castigo hacia sus cuerpos menstruantes. A partir de esta toma de consciencia, refieren, inician un movimiento transformador y reconciliador con sus cuerpos. Esta experiencia tendría tres posibilidades de ser vivida, cada una asociada a una de las tres posiciones feministas al interior del colectivo: despertar a la herida corporal, desde el feminismo chileno/occidental; enfrentar la herida corporal, desde el feminismo chileno/mapuche; y, reconocer la herida corporal colonial, desde el feminismo apegado a lo mapuche.

Despertar la herida corporal ilustra la posición de aquellas feministas que conciben el castigo corporal por el simple hecho de ser mujeres; desde este punto de vista, ellas entienden que su femineidad es causa de discriminación, tal como dice Laura: "La cultura y la propaganda y el condicionamiento social (...) habían hecho que yo viviera mi femineidad como un castigo". El castigo a lo femenino, usualmente transmitido por la madre, según comentan las mujeres, se ensaña en lo menstrual, que se concibe como feo y sucio, algo vergonzante que hay que ocultar. Al respecto, dice Camila: "Siempre hay que esconder la regla, por ejemplo, tener cuidado si uno mancha el baño, las sábanas". Desde este lugar, las mujeres experimentan un despertar a lo que llaman la herida corporal en la medida que despiertan de un estado de sometimiento anterior para constatar la violencia de la que han sido víctimas y el malestar vivido como herida corporal que han sufrido por ello. María José señala: "Había algo en mí que decía que había algo que no estaba funcionando bien, si yo tenía que usar toallitas, no conocer mi ciclo, que me doliera mucho (mi menstruación) (...) Yo sentía que eso no era algo positivo en mi cuerpo, entonces, dije, no, no quiero más".

Por su parte, enfrentar la herida corporal alude más que a una constatación del castigo sobre lo menstrual, a un deseo profundo de afrontar y, luego, sanar una herida corporal más holística, asociada a la femineidad indígena salvaje e inferior. Como dice Anita: "Puede ser como un llamado, algo así interior, como se quiera, pero yo lo siento así, como un llamado, que tenía que ver también con recuperarme..." Desde esta posición, las feministas chileno/mapuches reconocen la violencia y opresión sufridas como mujeres, pero no por ser mujeres, sino por ser mujeres mapuches, obligadas a silenciarse y a ocultarse por ello. Sobre ello, Anita señala: "Yo me reconozco también parte del mismo sistema (...) como sumiso de mujeres, como acalladas (...) Como que la mujer mapuche es oprimida en cierto aspecto poh (...) su vestimenta es un kupan, que es algo que va totalmente tapada". Este silenciamiento de la mujer mapuche es lo que las feministas de esta segunda posición se atreven a enfrentar en el Círculo. Sin embargo, ellas consideran que su silenciamiento como mujeres no ha implicado que su menstruación adquiera connotaciones de castigo. Al contrario, refieren que para ellas, menstruar denota una transformación de las mujeres mapuches durante ciertos días, como dice Gloria: "En ese momento las mujeres tienen un cambio". Menstruar es de este modo una característica esencial del cuerpo femenino y su ciclicidad.

Por último, reconocer la herida corporal colonial alude a aquellos significados de las feministas apegadas a lo mapuche donde no aparece la experiencia del castigo corporal por ser mujer ni por ser mujer mapuche, sino lo que aparece es un reconocimiento de la experiencia de castigo como sometimiento de los cuerpos de las mujeres mapuches en su relación con el gran sometimiento de su cultura. Al respecto, dice Ana: "Yo estoy convencida de que la herida trasciende, a los que están más arriba (...) y el que está más abajo, pero la herida es general, la violencia está en todo (...) la historia del poder siempre ha sido así, y esto es colonialismo". Desde esta posición, las mujeres tienen una experiencia consciente del castigo colonial que habría creado un cuerpo de mujeres mapuches "endurecido", ininteligible, oscuro, confuso y diluido en la corporalidad chilena. En torno a esto, la misma mujer señala: "Yo creo que igual todos estamos mediados por un proceso de colonización que (...) hay mujeres que pertenecen a familias muy violentadas y (...) por eso, reniegan todo tipo de características que puedan sobresalir y parecer mapuche". En este contexto, la menstruación aparece como un espacio colonizado, como Gina señala: "Respecto a quienes antes nos hablaban o nos decían las cosas (sobre la menstruación en la cultura mapuche), había momentos o personas indicadas (...) y creo que hubo un periodo también donde eso se desconoció, no se habló más".

Los significados que se producen desde estas tres posiciones coexisten en el Círculo, pero no establecen un diálogo igualitario entre sí. Esto es, las feministas chilenas/occidentales tienen dificultades para reconocer los discursos provenientes de los otros dos feminismos. Como dice Laura, feminista que podríamos ubicar en la posición chileno/occidental: "No siento la voz de la mujer mapuche hablando desde su experiencia", a lo que agrega que 
a las mujeres mapuches las ve trabajando en un proyecto político como pueblo, pero no luchando como mujeres. Al no reconocer los discursos de los otros dos feminismos, el feminismo chileno/occidental desconoce las prácticas de las mujeres mapuches, incluso las prácticas de sus propias compañeras del Círculo; como dice Laura, feminista chilena/occidental: "No podría definirlas (las prácticas corporales mapuches), más allá de la comida sana".

\section{Transformación del cuerpo castigado}

A partir de la toma de consciencia del castigo corporal, las participantes del Círculo refieren que experimentan una transformación de su cuerpo. Este momento también tendría para las participantes los siguientes tres sentidos significantes: manifestar la sanación desde lo social, para el caso del feminismo chileno/occidental, manifestar la sanación desde sí mismas, para el feminismo chileno/mapuche y, manifestar la sanación desde sus antepasadas, para el feminismo apegado a lo mapuche.

Sobre la manifestación de la sanación desde lo social, las feministas chilenas/occidentales dicen vivir una transformación íntegra del ser mujer por medio de "re significar la menstruación, no como suciedad, sino como una parte natural y sana de lo que es ser mujer", como dice Kathia. A partir de este cambio, las mujeres señalan que experimentan una reconciliación corporal, desde la cual se aproximan hacia el activismo social. La acción social es una forma de sanar, de reconocerse agentes de cambio, capaces de transgredir los significados peyorativos sobre el cuerpo femenino y de convocar a otras mujeres en esta lucha. Al respecto, la misma participante indica: "Sí, siento que hay activismo cuando las convocatorias o las invitaciones a nuestros encuentros o reuniones (como Círculo) son abiertas".

En cambio, desde la posición del feminismo chileno/mapuche, las mujeres manifiestan una sanación desde sí mismas, lo que quiere decir que se liberan respecto de las expectativas sociales occidentales conforme a las que han vivido y que las habían mantenido silenciadas como mujeres mapuches. Se trata de un proceso de curación personal que les permite regresar a una vida que consideran más saludable y más acorde a su pertenencia cultural. Como dice Anita: "Parte todo esto de uno sanarse si ha tenido algún trauma o herida (...) cuesta mucho porque es doloroso darse cuenta que vivo mi cuerpo según las expectativas del resto". Desde este lugar, la menstruación se convierte en un puente para reconectarse con lo mapuche, en específico, con sus raíces familiares matrilineales. Sobre este punto, Gloria comparte, orgullosa: "Con mi madre, con mis abuelas, sí, nosotros cuidábamos harto durante ese período, de no lavarnos, inclusive, de no andar en algunos espacios o hacer algunos trabajos...", actividades y ritos que no adquieren para ella un valor negativo, sino que expresan una posibilidad de rescatarse como mujer mapuche y de rescatar el lugar de las mujeres de su familia, en la historia de su pueblo.

Por último, desde el feminismo apegado a lo mapuche, la sanación se manifiesta desde la reconexión con las antepasadas y con la historia del pueblo mapuche. Las antepasadas o ancianas son valoradas y respetadas en su cultura porque son quienes otorgan conocimiento a las jóvenes; al traerlas al presente les permite nombrar y sanar su historia personal y como pueblo. En esta posición, las feministas cambian porque reconocen los dolores que las mujeres de su pueblo, incluyendo sus antecesoras, han sufrido. Ellas fueron violentadas, intelectual, moral y sexualmente, en un proceso que implicó lo que las integrantes del Círculo entienden como un "adormecimiento" de la dignidad y de la posibilidad de disfrutar como sujeto. Por esta razón, ubicadas en esta posición, las mujeres refieren sanarse a partir de conectarse con sus antepasadas y romper con el adormecimiento humano de un pueblo colonizado. Al respecto, dice Ana: "El placer, el goce cotidiano, como que ha vuelto a mí (...) ya desperté". Se trata de un proceso de reconexión corporal personal, que implica una resistencia de un pueblo frente a la violencia y la opresión occidental. En este contexto, la menstruación adquiere para las mujeres un sentido de resistencia anticolonial, es decir, la menstruación es una experiencia desde la cual revivir la dimensión espiritual del cuerpo. Tal como expresa una mujer mapuche, participante en el grupo de discusión: "Cuando mi mamá me explicó lo que me iba a suceder, cuando me iba a llegar mi primera menstruación, entonces, es parte de nuestro cuerpo (...) mi cuerpo es sagrado... era lo más sagrado nuestra menstruación...". Para la cultura mapuche, aunque se ha ido perdiendo con el tiempo, la menstruación es una bendición, es un momento sagrado manifestado en la tradición femenina mapuche del katan pilun, es decir romper las orejas y colocar aros como signo de fertilidad (GARCÍA; ROJAS, 2014).

Se trata de tres posiciones distintas, que tienen en común transformar un cuerpo castigado, pero en distintas dimensiones: como mujer, como mujer mapuche y como miembro de una cultura colonizada. La menstruación como castigo vivida por las participantes está sustentada desde la tradición judeocristiana como un justo castigo por la desobediencia 
a Dios (Lv 15, 19-33) hasta ser vista como enfermedad dolorosa, maldición (MORA, 2006; MONTECINO, 1984, CALFÍO, 2012; BACIGALUPO, 2013; CARREÑO, 2011). Los cuerpos de las mujeres mapuche fueron vinculados al mal o a lo kalku (MONTECINO, 1984) y con ello lo menstrual. A pesar de estas diferencias, las tres posiciones comparten una última condición: el hecho de construir estas significaciones en el Círculo, un espacio feminista entre mujeres y que les permite trascender sus castigos corporales.

\section{Trascendencia de lo corporal entre mujeres}

Las resignificaciones corporales de las mujeres feministas del Círculo tienen lugar en un espacio de transformación entre mujeres. Según su ex periencia, la compañía de otras hace posible las resignificaciones en cada una de las posiciones feministas al interior del colectivo. No sólo los significados sobre la menstruación y el cuerpo, racializado o no, se transforman para sanar a las mujeres, sino que ellas experimentan que se ubican más allá del castigo, lo cual se logra por medio de una variedad de prácticas reivindicadoras. Son tres los sentidos significantes que se producen al respecto: transcender en las prácticas, por parte del feminismo chileno/occidental; trascender en las prácticas y en la espiritualidad, por el feminismo chileno/mapuche; $y$, trascender en la espiritualidad y en la tierra, por el feminismo apegado a lo mapuche.

Sobre la trascendencia en las prácticas, las feministas de la posición chileno/occidental consideran que el castigo de ser mujeres puede superarse por medio del amor y la aceptación que proporcionan las compañeras del Círculo, o desde "desde la co-creación", como dice Kathia. Se trata de un proceso de co-resignificación corporal, centrado en la mujer menstruadora, que implica la resignificación de la menstruación a través de la producción de ciertas prácticas que, según refieren, están basadas en elementos como el uso del cinturón menstrual ${ }^{4}$, comida saludable ${ }^{5}$ y la medicina mapuche ${ }^{6}$. Al respecto, Kathia comenta: "Las mujeres mapuches también usan el cinturón, pero también lo usan como parte de la vestimenta, no como tan o quizás sí, pero no con tanto énfasis de que es protector energético de la matriz de la mujer". Este testimonio muestra, por un lado, que las mujeres estudiadas retoman elementos culturales mapuches como medios para su reconciliación corporal; y, por otro lado, de nueva cuenta, que desde esta posición las mujeres no observan que las mujeres mapuches sean parte de un proceso similar de reconciliación consigo mismas. Así lo comenta María Laura: "Siento que hay una distancia muy grande (de la mujer mapuche) con su cuerpo... Yo no las siento libres de todo y también de poca identificación con su esencia femenina, la mujer mapuche tiene que ser guerrera dura". Esencialismo, necesario de deconstruir, cimentado bajo el paradigma civilización-barbarie durante la invasión española y la república chilena donde se muestra a las mujeres mapuche como cuidadoras bravías de niños, ruka o casa y animales mientras los hombres combatían.

La posición de trascendencia en las prácticas y la espiritualidad, por otra parte, alude a la construcción de las feministas chileno/mapuches para transformarse por medio de la introspección y la autoreflexión, donde el colectivo actúa como un medio facilitador de este proceso. Como dice Anita: "Yo creo que es un trabajo interno (...) A veces se necesita como el colectivo (...) porque también es un camino, entonces siento que tú canalizas (en el colectivo), como que tú canalizas muchas cosas, (...) informaciones que así valoramos de otra manera". Desde esta posición, las feministas emprenden un cambio de trascendencia del castigo corporal por ser mujeres mapuches, que las lleva a reconectarse con sus raíces ancestrales por medio de realizar las mismas prácticas descritas para las feministas chileno/occidentales. Sin embargo, a diferencia de ellas, las feministas chileno/ mapuches les otorgan una dimensión espiritual, como muestra Anita: "Siento que la mujer mapuche, en cierto modo, tiene una espiritualidad mayor que la mujer eh winka"7. Desde esta espiritualidad, que vive lo cotidiano como con qué vestirse, las relaciones con la tierra, los alimentos y las personas, la mujer mapuche se reencuentra consigo misma y se ubica más allá del silenciamiento que la cultura chilena-occidental le ha impuesto a su corporalidad. Sin embargo, en esta posición, la trascendencia sigue teniendo su centro en la mujer menstruadora, como sujeto individual que se relaciona con lo occidental y lo mapuche. Lo anterior se observa en ciertas prácticas en las que coexisten ambas tradiciones, por ejemplo, cuando las mujeres se tiñen el cabello de morado u otro color llamativo, a la vez que visten sus trajes indígenas y participan de las ceremonias comunitarias como el Nguillatun ${ }^{8}$.

\footnotetext{
4 En mapudungun es trariwe, medio protector que se usa no solo en el tiempo de menstruar ayudando al equilibrio del cuerpo por el calor de la lana de oveja con el que es tejido.

5 Té de hierbas, maté, agua, frutas frescas y secas.

6 Uso de hierbas o lawen (remedio) del sistema médico mapuche: külon, foye. Además, con este fin son usados agua, barro, etc.

7 No mapuche.

${ }^{8}$ Principal ritual del pueblo mapuche para pedir, dar gracias, celebrar, reparar, etc.
} 
Por último, respecto del feminismo apegado a lo mapuche y su trascendencia en la espiritualidad y la tierra, esta categoría refiere a la experiencia de estas mujeres, de sentir que se ubican más allá del castigo corporal y colonial por medio de estrechar sus lazos con su comunidad y territorialidad. Con relación a esto, Ana menciona: "(la reconciliación) tiene mucho que ver con la historia familiar, y donde te tocó entre comillas vivir, o sea, si tu familia pertenece a Malleco o a Cautín (zonas con historia de colonialidad con excesiva violencia)". Estas mujeres también realizan las prácticas de trascendencia que efectúan las feministas desde las otras dos posiciones, pero a diferencia de ellas, su trascendencia no refiere a su superación de la experiencia de inferioridad como mujeres ni como mujeres mapuches, sino a su superación como sujetos individuales. Ellas transcienden en la medida que su cuerpo y su menstruación resisten la colonialidad, fortalecen su espiritualidad y se hacen parte de la tierra y la comunidad a la que pertenecen. Al respecto, la anterior participante señala: "(Siento) cómo cambia mi sentir corporal, por ejemplo, no hacia un hombre siempre sino que hacia algo más superior, puede ser, como conectado con la tierra".

Se han descrito tres procesos que dan cuenta de la experiencia de distintas mujeres, todas activistas, autoadscritas feministas del Círculo. Tomados en su conjunto, los tres procesos refieren a una pluralidad de significados en torno a lo menstrual, pero también a las limitaciones que al interior del colectivo las mujeres tienen para reconocerse entre sí. El diálogo intercultural es posible, porque las articulaciones entre lo occidental y lo mapuche coexisten y se retroalimentan en el mismo espacio. Sin embargo, las comprensiones mutuas son limitadas. ¿Qué sucede con las feministas chileno/occidentales respecto a su espiritualidad a partir del encuentro con las feministas apegadas a lo mapuche? ¿Cómo pueden las mujeres que ocupan la segunda y tercera posición hacer oír sus significados al interior del Círculo? ¿Cómo leen las feministas apegadas a lo mapuche la individualidad de las otras mujeres? Los resultados dan cuenta de un proceso de producción de significados múltiple, pero desconexo entre las tres posiciones feministas que componen el colectivo. No obstante, la pluralidad de experiencias sobre lo corporal y lo menstrual permiten pensar en la posibilidad de un diálogo intercultural, como se reflexiona en seguida.

\section{Discusión}

La investigación intentó describir los significados corporales sobre la menstruación que construyen las mujeres feministas del Círculo Hijas de la Luna y su relación con elementos de la cultura mapuche. Al respecto, los resultados revelaron que el activismo menstrual del colectivo implica una multiplicidad de significados que, empero, no parecen dialogar entre sí, a la vez que la posición mayormente heredera de la cultura occidental se impone sobre las otras. En este sentido, en el colectivo estudiado se estaría reeditando lo que Bobel (2010) identificó como apropiaciones de la cultura indígena que, despojada de su historicidad, corre el riesgo de convertirse en un nuevo instrumento de colonización.

En este último sentido, las mujeres del Círculo, cuando adoptan una posición dominante desde el feminismo chileno/occidental, reproducen la ceguera que ha caracterizado al feminismo occidental y al latinoamericano, que tienen una deuda histórica con las mujeres indígenas cuando no reconocen el castigo racializado de lo femenino en esta parte del mundo (HERNÁNDEZ, 2001; CURIEL; GALINDO, 2015). Desde este punto de vista, el cuerpo indígena sigue fuera de la reflexión y la reivindicación de las feministas menstruadoras.

Sin embargo, los hallazgos son prometedores porque muestran un aspecto diferencial del feminismo menstrual latinoamericano, representado por las posiciones feministas mapuche/chilenas y apegadas a lo mapuche. Estas últimas dejarían entrever resistencias, enunciadas desde lugares situados histórica y espacialmente, donde resisten significados sobre lo menstrual y lo corporal que se apartan de la visión cartesiana mente/cuerpo, considerando la interrelación en y entre las personas y el ambiente como principio básico de su cosmología y espiritualidad. Estos otros significados dan cuenta de la lucha histórica de las mujeres en Latinoamérica, por resistir la colonización de sus cuerpos, a la par que son herederas de la propia colonialidad que cuestionan. Los significados menstruales que producen dan cuenta de ambos procesos: una menstruación no peyorativa, sino natural y positiva, incluso sagrada (MORA, 2006; CARREÑO, 201 1; CALFÍO, 2012), que coexiste con otra experiencia menstrual producto de la colonialidad y de la construcción del cuerpo femenino como lo sucio e inmoral (DUSSEL, 2007).

Las producciones simbólicas de las feministas del Círculo dejan entrever caminos prometedores de reconciliación y liberación para las mujeres indígenas y mestizas. Su pluralidad permite ver la posibilidad de resignificar la corporalidad de las mujeres en la cultura occidental por medio de asumir la propia historia cultural. Esta última es el punto de partida para edificar una sanación corporal que esté libre de prejuicios raciales y violencias coloniales generacionales (Victoria PITTS, 2003; Andrea PERDOMO, 2013). Las feministas ape- 
gadas a lo mapuche y, en menor medida, las feministas mapuche/chilenas muestran las posibilidades de emprender este camino.

La relevancia del estudio se expresa teórica y prácticamente. En lo teórico, el estudio contribuye al cuestionamiento del feminismo occidental, en su expresión menstrual, sobre sus posibilidades de reconocer los cuerpos racializados de las mujeres. En lo práctico, los resultados contribuyen a repensar los estudios sobre el cuerpo de las mujeres y, desde allí, proponer intervenciones en salud acordes a sus realidades situadas histórica y espacialmente. Por último, ¿puede el cuerpo femenino comprenderse en términos de un diálogo de saberes? Esta es la pregunta que subyace en el estudio y que invita a que futuras investigaciones ahonden en las posibilidades de las feministas menstruadoras del sur para reconocerse mutuamente en sus diferencias.

\section{Referencias}

ALARCÓN, Miguel Ángel. "Algunas consideraciones antropológicas y religiosas alrededor de la menstruación". Revista Colombiana de Obstetricia y Ginecología, Colombia, v. 56, n. 1, p. 35-45, feb. 2005. Disponible en http://www.scielo.org.co/pdf/rcog/v56n1/v56n1 a05. pdf. Consultado el 30/05/2019.

BACIGALUPO, Ana Mariella. "Rituales de género para el orden cósmico: luchas chamánicas mapuche por la totalidad". Revista de Antropología, Santiago de Chile, v. 17, p. 4774, 2003-2004. Disponible en https://revistadeantropologia.uchile.cl/index.php/RCA/article/ view/17353. Consultado el 30/05/2019.

BACIGALUPO, Ana Mariella. "Las mujeres machi en el siglo XX-XXI: ¿personificación de la tradición o desafío a las normas de género?". In: STUVEN, Ana María; FERNANDOIS, Joaquín. Historia de las mujeres en Chile. Tomo Il. Chile: Editorial Taurus, 2013. p. 433-502.

BOBEL, Chris. "Our revolution has style: contemporary menstrual product activists "doing feminism"" (En línea). 2010. Disponible en blogspot http://menstruar.blogspot.cl/2010/10/ breve-historia-del-activismo-menstrual.html. Consultado el 22/11/2016.

BUTLER, Judith. Cuerpos que importan: sobre los límites materiales y discursivos del "sexo". Buenos Aires. Argentina: Editorial Paidós, 2002.

CALFíO, Margarita. "Peküyen". In: NAHUELPAN, Héctor; HUINCA, Herson; MARIMAN, Pablo. TA IÑ FIJKE XIPA RAKIZUAMELUWÜN. Historia, colonialismo y resistencia desde el país mapuche. Temuco: Ediciones Comunidad de Historia Mapuche, 2012. p. 279-298.

CARREÑO, Ximena. Rituales femeninos, cuerpo fluido menstrual sembrando la luna. 2011. Pregrado (Programa Licenciada en Artes Visuales) - Facultad de Artes de la Universidad de Cuenca, Cuenca, Provincia de Cuenca, España.

CURIEL, Ochy; GALINDO, María. Descolonización y despatriarcalización de y desde los feminismos de AbyaYala. España: Editorial ACSUR- LAS SEGOVIAS, 2015.

CEPAL. Los Pueblos indígenas en América Latina. 2014. Disponible en https://repositorio.cepal.org/bitstream/handle/11362/37222/S1420521_es.pdf. Consultado el 30/05/2019.

CRESWELL, John. Diseño de investigación. Métodos cualitativos, cuantitativos y métodos mixtos. California: Ediciones SAGE, 2014.

DEBRA, Merskin. "New blood: third-wave feminism and the politics of menstruation". Contemporary Sociology: a journal of reviews, v. 40, n. 4, p. 435-437, jul. 201 1. Disponible en https://journals.sagepub.com/doi/10.1177/0094306111412516g. Consultado el 30/05/2019.

DE VALLESCAR, Diana. Cultura, multiculturalismo e interculturalidad. Hacia una racionalidad intercultural. Madrid. España: Editorial Covarrubias, 2010.

DESCARTES, René. Meditaciones metafísicas. Santiago. Chile: Editorial Universitaria, 1641.

DUSSEL, Enrique. Para una erótica latinoamericana. Caracas. Venezuela: Fundación Editorial El perro y La rana, 2007.

ESTEBAN, Mari Luz. "Cuerpos y políticas feministas: el feminismo como cuerpo". In: VILLALBA, Cristina; ALVAREZ, Nacho. Cuerpos politicos y agencia. España: Universidad de Granada, 2011. p. $45-84$. 
FELITTI, Karina. "El tabú de la menstruación: sangre azul". Revista Anfibia (En línea). Universidad Nacional de San Martín, Argentina, 2015. ISSN 2344-9365. Disponible en http://www. revistaanfibia.com/ensayo/sangre-azul/. Consultado el 10/10/2016.

FORNET-BETANCOURT, Raúl. Notas sobre el sentido de la pregunta por una filosofía americana y su contexto histórico-cultural. In: SEMINARIO DE HISTORIA DE LA FILOSOFÍA ESPAÑOLA, V, 1988, Salamanca, Universidad de Salamanca. Actas. Salamanca: Ediciones Universidad de Salamanca, 1988. p. 437-445.

GADAMER, Hans-Georg. Verdad y método. Salamanca. España: Ediciones Paidós, 1997.

GARCÍA, Loreto; ROJAS, Soledad. Mujeres mapuche y domo kimün. 2014. Pregrado (Programa de Sociología) - Universidad Diego Portales, Santiago, RM, Chile.

GUILLO, Miren. "La in-corporación de la investigación: políticas de la menstruación y cuerpos (re) productivos". Revista Nómadas. Universidad Central Colombia, n. 39, p. 233245, oct. 2013. Disponible en http://www.scielo.org.co/scielo.php?script=sci_abstract\&pid=S0121-75502013000200016\&lng=en\&nrm=iso\&tIng=es. Consultado el 30/05/2019.

HARDING, Sandra. Ciencia y feminismo. Madrid: Ediciones Morata, 1996.

HERNÁNDEZ, Aida. "Entre el etnocentrismo feminista y el esencialismo étnico. Las mujeres indígenas y sus demandas de género". Debate feminista, Ciudad de México, v. 24, p. 206229, oct. 2001. Disponible en http://www.debatefeminista.pueg.unam.mx/wp-content/ uploads/2016/03/articulos/024_13.pdf. Consultado el 05/10/ 2015.

HIDALGO, Jorge; CASTRO, Nelson. "Género, etnicidad, poder e historia indígena en Chile". In: STUVEN, Ana María; FERNANDOIS, Joaquín. Historia de las mujeres en Chile. Tomo I. Chile: Editorial Taurus, 2010. p 83-122.

HURTADO, María de la Luz. "Cuerpo y mujer chilena en la urbe ilustrada del siglo XIX". In: STUVEN, Ana María; FERNANDOIS, Joaquín. Historia de las mujeres en Chile. Tomo I. Chile: Editorial Taurus, 2010. p. 375-425.

KELLE, Udo. "¿Hacer "emerger" o "forzar" los datos empíricos? Un problema crucial de la teoría fundamentada reconsiderada". Revista FQS (En línea). Berlín, v. 6, n. 2, 2005, Art 27. Disponible en http://www.qualitative-research.net/index.php/fqs/article/view/467/3397 Consultado el 05/02/2016.

KETTERER, Lucy. "Mujeres y política. Notas acerca del movimiento de mujeres en la región de la Araucanía de Chile". Revista La Aljaba (En línea). Luján - Argentina, v. 15, ene/dic. 2011. Disponible en http://www.cervantesvirtual.com/descargaPdf/mujeres-y-politica-notas-acerca-del-movimiento-de-mujeres-en-la-region-de-la-araucania-de-chile-886468/. Consultado el 20/05/2019.

KIRKWOOD, Julieta. Ser política en Chile: las feministas y los partidos. Santiago, Chile. Facultad Latinoamericana de Ciencias Sociales: Ediciones FLACSO, 1986.

LAMAS, Marta. "Diferencias de sexo, género y diferencia sexual". Revista Cuicuilco, México, v. 7, n. 18, ene/abr. 2000. Disponible en https://hum.unne.edu.ar/generoysex/seminariol/ s1_08.pdf. Consultado el 20/05/2019.

LEVIL, Ximena. "Reflexiones y relatos de una mujer mapuche". In: BOITANO, Angela; RAMM, Alejandra. Rupturas e Identidades. Santiago. Chile: Editorial Ril. 2015. p. 29-43.

LEÓN, Orfelio; MONTERO, Ignacio. Métodos de investigación en Psicología y Educación. Las tradiciones cuantitativa y cualitativa. Cuarta edición. Facultad de Psicología Universidad Autónoma de Madrid. España, 2015.

LUGONES, María. "Colonialidad y género". Revista Tabula Rasa, Bogotá -Colombia n. 9, p. 73-101, jul/dic. 2008. Disponible en https://www.revistatabularasa.org/numero-9/05lugones. pdf. Consultado el 25/05/2019.

MAMANI, Vicenta. Identidad y espiritualidad de la mujer aymara. Cochabamba. Bolivia: Editorial Itinerarios, 2014. 
MERLEAU-PONTY, Maurice. Phénoménologie de la perception. Paris: Ediciones Gallimard, 1945. (Trad. Cast. de CABANES, J. (1975). Fenomenología de la percepción. Barcelona: Península).

MONTECINO, Sonia. Mujeres de la tierra. Santiago. Chile: Ediciones CEM, 1984.

MORA, Claudia; RíOS, Marcela. "¿De política de representación a política de coalición? Posibilidades de movilización en el Chile pos-dictadura". Revista Polis, Santiago, v. 8, n. 24, p. 133-145, 2009. Disponible en https://scielo.conicyt.cl/scielo.php?script=sci_arttext\&pid=S0718-65682009000300008. Consultado el 15/05/2019.

MORA, Ziley. Magia y secretos de la mujer mapuche. Sexualidad y sabiduría ancestral. Temuco. Chile: Editorial Uqbar, 2006.

OLIVA, Asunción. "Hacia una ontología social del cuerpo en Buthler: análisis y límites". Investigaciones Feministas (En línea). Madrid, v. 6, p. 85-107, 2015. Disponible en http://revistas. ucm.es/index.php/INFE/article/view/51381. https://doi.org/10.5209/rev_INFE.2015.v6.51381. Consultado el 12/02/2016.

PALESTRO, Sandra. "Mujeres en movimiento 1973-1989". Estudios Sociales, n. 14, FLACSO, Santiago - Chile, 1991. Disponible en memoriachilena.gob.cl/602/w3-article-67282.html. Consultado el 22/05/2019.

PAREDES, Julieta. Hilando fino desde el feminismo comunitario. México: Editorial El Reboso, 2013.

PERDOMO, Andrea et al. "Prácticas corporales acerca del cuidado de sí en la comunidad indígena Sikuani". Revista Facultad de Medicina, Bogotá, v. 61, n. 4, p. 381-384, oct. 2013. Disponible en http://www.scielo.org.co/pdf/rfmun/v61n4/v61n4a7.pdf. Consultado el 30/05/2019.

PITTS, Victoria. In the flesch. The cultural politics of body modification. p. 1-23. 2003.

SALAZAR, Cecilia. "Pueblo de humanos: metáforas corporales y diferenciación social indígena en Bolivia". Revista Anthropologica, La Paz, v. 24, p. 5-27, dic. 2006. Disponible en http://www.scielo.org.pe/scielo.php?pid=S0254-92122006000100001 \&script=sci_abstract. Consultado el 30/05/2019.

SEGATO, Rita. "Género y colonialidad: en busca de claves de lectura y de un vocabulario estratégico descolonial" In: QUIJANO, Aníbal; MEJÍA, Julio. La cuestión descolonial. Lima: Universidad Ricardo Palma - Cátedra América Latina y la colonialidad del poder, 2010. p. 27-59.

SOENGAS, Stella; ZAMORANO, Silvia. El cuerpo en la posmodernidad. In: CONGRESO INTERNACIONAL DE INVESTIGACIÓN Y PRÁCTICA PROFESIONAL EN PSICOLOGÍA, l; JORNADAS DE INVESTIGACIÓN, XVI; ENCUENTRO DE INVESTIGADORES EN PSICOLOGÍA DEL MERCOSUR, V, 2009, Buenos Aires, Facultad de Psicología de la Universidad de Buenos Aires. Acta académica, 2009, p. 334- 336. Disponible en https://www.aacademica.org/000-020/717. Consultado el 07/02/2017.

WALSH, Catherine. Interculturalidad crítica y (de)colonialidad. Ensayos desde Abya Yala. Ecuador: Ediciones Abya Yala, 2012.

Andrea de Lourdes Castillo-Muñoz (acastillo@uct.cl) es teóloga (Pontificia Universidad Católica de Chile), Magíster en Estudios Interculturales (Universidad Católica de Temuco) y doctoranda en Estudios Inerculturales (Universidad Católica de Temuco). Línea de investigación: Género, espiritualidad e interculturalidad.

Gloria Mora-Guerrero (gmora@uct.cl) es psicóloga (Universidad de Guanajuato), Magíster en Psicología Comunitaria (Universidad de Chile) y Doctora en Estudios Americanos (Universidad de Santiago de Chile). Profesora e investigadora de la Universidad Católica de Temuco. Línea de investigación: Género, organización social del cuidado y asociacionismo productivo de mujeres rurales en la Araucanía. 


\section{COMO CITAR ESTE ARTÍCULO, DE ACUERDO CON LAS NORMAS DE LA REVISTA}

CASTILLO-MUÑOZ, Andrea de Lourdes; MORA-GUERRERO, Gloria. "¿Pensar el cuerpo femenino como diálogo de saberes?". Revista Estudos Feministas, Florianópolis, v. 29, n. 1, e65893, 2021.

\section{CONTRIBUCIÓN DE AUTORÍA}

Andrea de Lourdes Castillo-Muñoz: concepción, recolección de datos y análisis de datos, elaboración del manuscrito.

Gloria Mora-Guerrero: análisis de datos, correção del manuscrito, redacción, discusión de resultados.

\section{FINANCIACIÓN}

Vicerrectoría de Investigación y Postgrado de la Universidad Católica de Temuco, VIPUCT № 2016GI$-\mathrm{MB}-02$

\section{CONSENTIMIENTO DE USO DE IMAGEN}

No se aplica.

\section{APROBACIÓN DE COMITÉ DE ÉTICA EN INVESTIGACIÓN}

Todos los entrevistados firmaron el Consentimiento Informado de acuerdo con las directrices de la investigación en curso en la Universidad Católica de Temuco durante la recolección de datos.

\section{CONFLICTO DE INTERESES}

No se aplica.

\section{LICENCIA DE USO}

Este artículo tiene la licencia Creative Commons License CC-BY 4.0 International. Con esta licencia puedes compartir, adaptar, crear para cualquier finalidad, siempre y cuando cedas la autoría de la obra.

\section{HISTORIAL}

Recibido el 25/06/2019

Presentado nuevamente el 25/10/2020

Aprobado el 16/11/2020

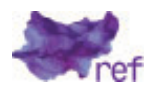

